\title{
Recent Pharma News
}

\section{FDA Approves New Drug for Heart Failure}

Entresto was granted 'fast track' approval by the agency.

THURSDAY, July 9, 2015

HealthDay News -- The U.S. Food and Drug Administration has approved the first in a new class of drugs that show promise for combating heart failure.

The approval of Entresto (sacubitril/varsatan) was sped up after a clinical trial found it significantly reduced the rate of death and hospitalization due to heart failure compared to another, often-prescribed treatment, the FDA said in a news release.

Heart failure, which is characterized by the heart's inability to pump enough blood, affects some 5.1 million people in the United States. Heart attack, high blood pressure and other conditions that damage the heart are primary causes, the FDA said.

"Heart failure is a leading cause of death and disability in adults," Dr. Norman Stockbridge, director of the division of cardiovascular and renal products in the FDA's Center for Drug Evaluation and Research, said in the news release. "Treatment can help people with heart failure live longer and enjoy more active lives."

Entresto is the first in a new class of drugs called angiotensin receptor neprilysin inhibitors, or ARNIs.

The new drug was evaluated in clinical trials involving more than 8,000 adults. It was shown to reduce the rate of cardiovascular death and hospitalization. Most trial participants also took standard heart failure medications, such as beta blockers and diuretics.

Common side effects of Entresto included low blood pressure, elevated blood potassium and impaired kidney function, according to the FDA.

A more serious adverse effect was angioedema, an allergic reaction characterized by swelling of the lips or face. Blacks and people with a history of angioedema are at higher risk of this reaction, the FDA said. People taking the drug should seek immediate medical help if they develop facial swelling or trouble breathing, the agency said.

Entresto should not be used with any ACE inhibitor drug, which would increase a user's risk of angioedema.
And women should discontinue Entresto as soon as possible if they become pregnant, the FDA warned.

The drug is made by Novartis Pharmaceuticals, based in East Hanover, N.J.

\section{FDA Strengthens Heart Attack, Stroke Warning for Popular Painkillers}

NSAIDS include such drugs as ibuprofen and naproxen; aspirin not included in alert.

Thursday, July 9, 2015

HealthDay News -- The U.S Food and Drug Administration on Thursday strengthened the warning labels for widely used painkillers like ibuprofen and naproxen, saying they can increase the risk of heart attack or stroke.

The FDA is asking people to think carefully about their use of nonsteroidal anti-inflammatory drugs (NSAIDs), particularly if they've already had a heart attack, according to a consumer update on the agency's website.

The agency said it is taking this action based on recent data that shows the risk of heart attack or stroke can increase even after using NSAIDs for a short time.

"They used to say they might cause risk of heart attack or stroke. Now we are saying they do cause increased risk of heart attack and stroke," FDA spokesman Eric Pahon told NBC News.

In particular, people should avoid taking multiple products that contain NSAIDs, according to the revised FDA warning.

Common over-the-counter NSAIDs include ibuprofen (Motrin, Advil) and naproxen (Aleve), but NSAIDs also can show up in combination medicines like multisymptom cold products.

"Be careful not to take more than one product that contains an NSAID at a time," Dr. Karen Mahoney, deputy director of the FDA's Division of Nonprescription Drug Products, said in the agency's announcement. People should check the list of active ingredients in the drug facts label if they aren't sure whether a product contains an NSAID, she said. 
Although aspirin is also an NSAID, the revised warning doesn't apply to aspirin, the FDA said.

The agency will require drug manufacturers to include the updated warning on both prescription and over-the-counter brands of NSAIDs. Over-the-counter NSAIDS are generally used to treat pain, inflammation and fever, while the stronger prescription brands are reserved for chronic and debilitating conditions like arthritis.

People with heart disease or high blood pressure should consult a doctor before using an NSAID, the FDA said.

However, the agency noted that the cardiovascular risk also is present in people without heart health problems. "Everyone may be at risk -- even people without an underlying risk for cardiovascular disease," said Dr. Judy Racoosin, deputy director of the FDA's Division of Anesthesia, Analgesia and Addiction Products.

The FDA first added a boxed warning to NSAID labels for the cardiovascular risk in 2005, after Merck \& Co. pulled its popular pain reliever Vioxx off the market the year before. Vioxx, an NSAID, had been linked to heart attack and stroke.

Current labeling on over-the-counter NSAIDs warns patients to take the lowest dose possible for the least amount of time possible, and to not use them to treat pain for longer than 10 days.

"These medicines have a long history of safety and efficacy when used as directed," the Consumer Healthcare Products Association, which represents nonprescription drug makers, said in a statement to the Associated Press. The group said it would cooperate with the FDA as it requests updates to the labels.

SOURCES: U.S. Food and Drug Administration, news release, July 9, 2015; NBC News; Associated Press

\section{Uncontrolled Diabetes May Boost Dementia Risk}

Thursday, July 9, 2015

HealthDay News -- Diabetes patients with high rates of complications from the disease may face increased risk for dementia, a new study suggests.
"We found that as diabetes progresses and an individual experiences more complications from the disease, the risk of dementia rises as well," wrote Dr. Wei-

Che Chiu, of the National Taiwan University College of Public Health, in Taipei.

Better blood sugar control can help prevent the mental decline associated with diabetes, he and his colleagues said.

They examined data from more than 431,000 people in Taiwan who were older than 50 and newly diagnosed with diabetes.

Complications of diabetes include vision loss, kidney failure and nerve damage.

Over 12 years of follow-up, more than 6 percent of the patients were diagnosed with dementia. Those with a greater number of diabetes complications were at higher risk for mental decline than those with few or no complications.

The study was published online July 9 in the Journal of Clinical Endocrinology \& Metabolism.

"The study demonstrates why it is so crucial for people with diabetes to work closely with health care providers on controlling their blood sugar. Managing the disease can help prevent the onset of dementia later in life," Chiu said in a journal news release.

More than 29 million Americans have diabetes, according to the Endocrine Society. Of every 100 Americans with the disease, 21 have nerve damage, 27 have kidney damage and 29 to 33 have eye damage.

SOURCE: Journal of Clinical Endocrinology \& Metabolism, news release, July 9, 2015

\section{Lifestyle and Heart Failure Risk}

Wednesday, July 8, 2015

HealthDay News -- Making the right lifestyle choices can significantly cut your risk of heart failure, according to a new study.

Researchers followed nearly 4,500 men and women for about two decades tracking their diet, walking and exercise habits, alcohol use, smoking status, height and weight, waist circumference and heart health. During the monitoring period, nearly 1,400 cases of heart failure were documented. The findings showed that adults who walked at a faster pace had a lower risk. Enjoying leisure activities 
that burned 845 calories or more per week, not smoking, modest alcohol consumption and avoiding obesity were also associated with reduced rates of heart failure. Participants who scored four or more healthy behaviors were about half as likely to have heart failure as those with zero or one low-risk factor. The researchers found no relationship between dietary patterns and heart failure and exercise intensity was not as important as walking pace. The lead author says it's encouraging to learn that simple changes can help reduce risk of heart failure and emphasizes that eating a healthy diet is still critical for preventing other cardiovascular disease and type 2 diabetes.

http://www.nlm.nih.gov/medlineplus/videos/news/Life_St yle_070715.html

\section{Type 2 Diabetes May Damage Thinking Skills: Study}

Test performance declined noticeably in just two years.

Wednesday, July 8, 2015

HealthDay News -- In as little as two years, people with type 2 diabetes may develop problems with blood flow in the brain, which could lower their thinking and memory skills, a small study suggests.

"Our major finding is we have linked the acceleration of the cognitive decline to impaired blood flow regulation in the brain," said senior study author Dr. Vera Novak, an associate professor of neurology at Harvard Medical School in Boston.

The problem the researchers found was with dilation of the blood vessels, which allows more blood to flow through the brain. Adequate amounts of blood are crucial for thinking skills and other activities.

The researchers found that the higher someone's average blood sugar levels were over the previous several months (a measure called A1C), the worse the problem with blood vessel dilation was, Novak said.

The study was published online July 8 in the journal Neurology. It was funded by the U.S. National Institute on Aging, the American Diabetes Association, the Harvard Clinical and Translational Science Center and the U.S. National Center for Research Resources.

In the study, the researchers evaluated 40 people. Their average age was 66. Nineteen of the study volunteers had type 2 diabetes, and 21 didn't have the blood sugar disease.

In type 2 diabetes, the body doesn't use insulin efficiently and eventually can't make enough insulin to control blood sugar levels, according to the American Diabetes Association (ADA). Insulin is a hormone that's crucial for metabolizing carbohydrates in foods. More than 29 million people in the United States have diabetes, and most of them have type 2 diabetes, the ADA said.

The researchers tested everyone at the start of the study, and again two years later. The volunteers completed thinking and memory tests. They were also given MRI scans to look at blood flow in their brains, and they had blood tests to measure their average blood sugar levels and inflammation.

At the two-year mark, those with type 2 diabetes had less ability to regulate blood flow to the brain when needed and scored lower on the thinking and memory tests.

On one test that looked at learning and memory, the scores of those with diabetes dipped an average of 12 percent, from 46 points to 41 . Those without type 2 diabetes stayed at an average of 55 points over the two years.

A decline from 46 to 41 would translate roughly to remembering 10 words on a memory test the first time, and then remembering only 8 or 9 two years later, said Novak. "It's only [in] two years, that's what is concerning."

The higher the inflammation levels, the worse the blood flow regulation, the research team found. That was true even for people who had good control of their diabetes.

Blood flow regulation decreased 65 percent in the people with type 2 diabetes, the researchers found.

Dr. Marc Gordon, chief of neurology at Zucker Hillside Hospital, North Shore Long Island Jewish Health System, Manhasset, said it's not new to suggest type 2 diabetes is linked with inflammation and stress to the cells that can lead to problems in the blood vessels.

"What's new here is they are documenting that the changes in the blood vessel in response to circumstances is what seems to be predicting a decline in cognition," said Gordon, who is also professor of neurology and psychiatry 
at Hofstra North Shore Long Island Jewish School of Medicine.

In other words, he said, it appears the inability of the blood vessels to respond to various demands is what leads to the thinking problem, although inflammation also plays a role in damaging the blood vessels.

In previous research, Novak found that the brain of a person with diabetes is about five years older, on average, than the brain of someone without the condition. "So essentially, the diabetic brain ages faster," she said.

The researchers added that a study with a larger group of people and done for a longer time is needed to better understand how type 2 diabetes may affect blood flow to the brain.

\section{Echinacea Preparation as Effective as Tamiflu ${ }^{\circledR}$ in Early Flu Cases in Large Clinical Trial}

June 17, 2015

New clinical research suggests that an herbal medicinal product containing a proprietary combination of a concentrated echinacea herb and root extract is as effective as the conventional antiviral medicine oseltamivir (Tamiflu ${ }^{\circledR}$ ) in the early treatment of influenza. The results of the randomized, double-blind, controlled clinical trial were published online in April in the openaccess journal Current Therapeutic Research.

For the study, researchers recruited 473 patients who had exhibited flu symptoms for less than 48 hours from 29 primary care practices in the Czech Republic. The patients were randomly assigned to take Echinaforce ${ }^{\circledR}$ Hotdrink syrup, a beverage containing an alcoholic extract prepared from freshly harvested echinacea (Echinacea purpurea)herb and root (95\% herb; 5\% root) supplemented with European elderberry (Sambucusnigra), for 10 days, or oseltamivir for five days followed by placebo for five days. (EchinaforceHotdrink is produced and marketed by A. Vogel Bioforce AG of Roggwil, Switzerland. It is not currently sold in the United States.)

The primary endpoint of the clinical trial was the percentage of patients with mild or no symptoms after one, five, and 10 days of treatment. At each time point, the researchers found that a similar number of patients had recovered in both groups. After day one, $1.5 \%$ of patients in the EchinaforceHotdrink group and $4.1 \%$ of those in the oseltamivir group exhibited mild or no symptoms; recovery rates for days five and 10 were $50.2 \%$ and $48.8 \%$, and $90.1 \%$ and $84.8 \%$, respectively.

Statistical analyses revealed that the two treatments were, in fact, non-inferior to each other; that is, they were approximately equal in their therapeutic effects. When the researchers looked at a subgroup of patients with laboratory-confirmed influenza (as opposed to those with a clinical flu diagnosis), recovery rates still did not vary between the two actives. Finally, there was a trend towards a higher proportion of recovered patients after 10 days of treatment with EchinaforceHotdrink.

"This is yet another significant human clinical trial that helps to document the clinical benefits of this specific Swiss echinacea extract," said Mark Blumenthal, founder and executive director of the American Botanical Council, a leading nonprofit research and education organization.

"The size of this trial -473 patients - is one of the largest echinacea clinical trials ever published," added Blumenthal, who noted that the largest echinacea clinical trial in the scientific literature - with 755 subjects — was conducted with the same Echinaforce extract, and showed a preventive effect against respiratory tract infections.

"It is rewarding to see an herbal products manufacturer investing resources in a well-designed clinical study on a phytotherapeutic product," noted Stefan Gafner, $\mathrm{PhD}$, ABC's chief science officer. "The combination of echinacea and European elderberry has resulted in the same treatment outcome as the standard drug oseltamivir in the study population, providing evidence for the safety and efficacy of this product."

In the new clinical trial, complications were limited in both groups; $6.5 \%$ of patients in the oseltamivir group experienced respiratory-related issues such as pneumonia, bronchitis, or sinusitis, and gastrointestinal complications. An insignificantly less number of patients in the Echinaforce group (2.5\%) experienced respiratory, but no gastrointestinal, complications. Researchers determined that 10 adverse events were related to the treatments four events in the Echinaforce group (1.7\%) and six events in the oseltamivir group (2.2\%), an insignificant difference. Patients taking oseltamivir were more likely to experience gastrointestinal issues such as nausea and vomiting. 
There were also no statistically significant differences between groups in terms of antibiotic use, intermediate doctor visits, use of over-the-counter medications for symptoms, "ability to return to normal daily activities," or physician- and patient-reported efficacy of the treatments.

"EchinaforceHotdrink has here been demonstrated as attractive therapy for acute influenza treatment with better safety and comparable efficacy profile to the neuraminidase inhibitor Oseltamivir," the authors

Information collected and compiled by:

Mr. Md. Akbar Hossain

Assistant Professor, Department of Pharmacy

Dhaka International University, Dhaka concluded. "Its availability as over-the-counter medicine allows for a very early treatment start, which is central for treatment success with any intervention. Further studies are warranted.'

$\mathrm{ABC}$ members and registered users of the $\mathrm{ABC}$ website can access the more detailed ABC HerbClip summary of the study - available here — on ABC's website (www.herbalgram.org). 